\title{
Differential Profile of Proinflammatory/Proresolving Lipid Mediators in Acute Inflammation Model using Young and Old Skin Biopsies
}

\author{
Lopez-Gaydon Amandine', Baillif Vincent ${ }^{2}$, Bertholon Cindy', Van Goethem Emeline ${ }^{2}$, Demarne \\ Frédéric ${ }^{1}$, Dubourdeau Marc ${ }^{2}$ and Bechetoille Nicolas ${ }^{* 1}$
}

${ }^{1}$ Gattefossé, RひD Department, FR-69804, Saint-Priest, France.

*Correspondence:

Nicolas Bechetoille, Gattefossé, R\&D Department, FR-69804, Saint-Priest, France, Tel. +33 472229 800; E-mail: nbechetoille@

${ }^{2}$ Ambiotis, 3 Rue des Satellites, FR-31400, Toulouse, France. gattefosse.com.

Received: 08 February 2019; Accepted: 14 March 2019

Citation: Lopez-Gaydon Amandine, Baillif Vincent, Bertholon Cindy, et al. Differential Profile of Proinflammatory/Proresolving Lipid Mediators in Acute Inflammation Model using Young and Old Skin Biopsies. Dermatol Res. 2019; 1(1); 1-2.

To the Editor,

Bioactive lipid mediators derived from polyunsaturated fatty acids (PUFA) contribute to skin health through a fine and a spatiotemporal control of inflammation and immune response [1]. Arachidonic acid (AA; 20:4n-6), eicosapentaenoic acid (EPA; 20:5n-3) and docosahexaenoic acid (DHA; 22:6n-3) are the most predominant essential fatty acids in human skin [2]. They are precursors for synthesis of mediators that are pro-inflammatory or that actively end this process and named for this specific effect, specialized proresolving lipid mediators (SPMs). Upon induction of inflammation resolution, cells that initially triggered inflammation undergo a biochemical paradigm shift, known as "lipid mediator class switch", during which they stop producing classical proinflammatory mediators and start to actively synthesize SPMs. These include AA-derived lipoxins (LXA4 and LXB4), EPA-derived resolvins and DHA-derived resolvins, protectins and maresins [3].

It has been shown that failure to engage proresolving pathways prolongs chronic inflammation [4] and that dysregulation of bioactive lipid metabolism occurs in several chronic inflammatory skin diseases [1]. Moreover, ageing is associated with an overt inflammatory phenotype increasing chronic inflammatory disease pathogenesis and prevalence in elderly [5]. Age-associated alterations in inflammation and resolution programs were recently reported in aged mice [6]. All this is warranting the hypothesis that inflammation and its resolution are impaired in aged human skin. Skin explants from young $(16,23,34$ years [24 \pm 9$])$ vs. old donors $(58,58,61$ years $[59 \pm 2])$ were obtained with informed consent of patients undergoing surgical discard, in accordance with the ethical guidelines of the Tissue Bank Institute of Edouard Herriot Hospital (Lyon, France). Ex vivo skin explants were cultured in Snapwell ${ }^{\mathrm{TM}}$ inserts (Corning) at $37^{\circ} \mathrm{C}$ in $5 \% \mathrm{CO}_{2}$, at the airliquid interface, in Prime-3D medium (CnT-PR-3D, CELLnTEC) supplemented with normocin ${ }^{\mathrm{TM}}$ (Invivogen). Ex vivo skin explants were topically treated with $50 \mu \mathrm{l}$ of a gel formulation containing phorbol myristate acetate (PMA) 1.5\% (Sigma) for 2, 4, 8, 24 and 48 hours. Ex vivo skin explants treated with a PMA-free formulation served as control. At each culture time-point, ex vivo skin explants were harvested, frozen in liquid nitrogen and stored at $-80^{\circ} \mathrm{C}$ until quantification of bioactive lipids. Metabololipidomic study used a procedure adapted from Le Faouder et al. [7]. Briefly, ex vivo skin explants were lysed and solid phase extraction was performed to extract bioactive lipids. Then, LC-MS/MS analysis used the UHPLC system (Agilent LC1290 Infinity) coupled to Agilent 6490 triple quadrupole MS (Agilent Technologies) equipped with electro-spray ionization operating in negative mode. Results were expressed as quantity of skin-tissue lipids $(\mu \mathrm{g} / \mathrm{mg})$. The area-underthe-curve (AUC) was calculated using the trapezoidal rule. AUCs underwent principal component analysis (PCA) using TANAGRA software. Data were expressed as mean \pm standard error of the mean (SEM) from 3 independent experiments.

Analysis of 24 lipid mediators (Figure 1A) has shown a strong mobilization of AA pathway with subsequent biosynthesis of proinflammatory (PGE2, thromboxane B2) and proresolving mediators (LXA4, LXB4) in young skin. By contrast, EPA and DHA pathways were preferentially mobilized in old skin. However, despite the production of 14-HDOHE, 17-HDOHE and 18-HEPE (which are the precursors of resolvins, protectins and maresins) no SPMs issued from these DHA and EPA precursors could be detected. This may suggest that SPMs are either produced at undetectable levels or are not produced in old skin, indicating then a failed engagement of proresolving signalling. 
A

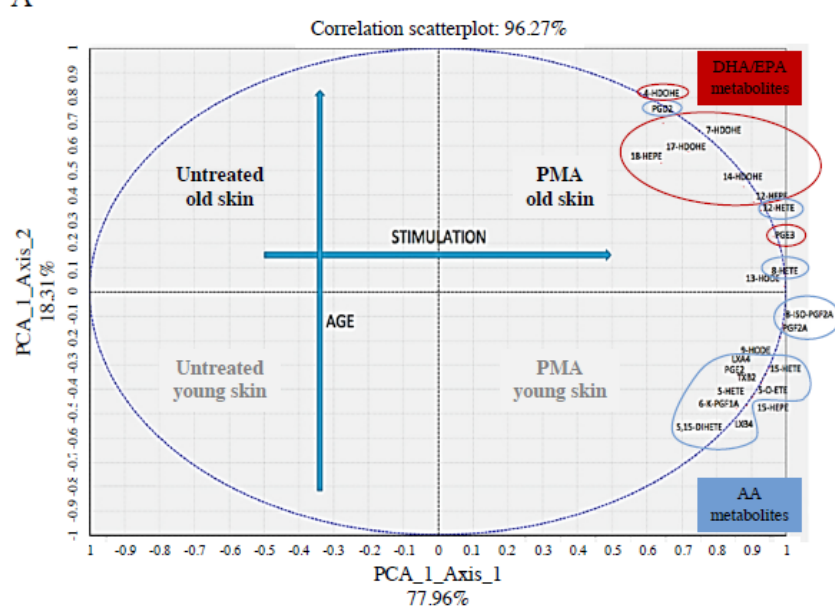

Comparative metabololipidomic profiling of bioactive lipid mediators in young vs. old PMA-challenged $e x$ vivo skin explants. Quantification of 24 lipid mediators over time $(0,2,4,8,24$ and $48 \mathrm{~h})$ by LC/MS-MS and AUCs calculation followed by principal component analysis (A). Production of $\mathrm{PGE}_{2}(\mathrm{~B})$ and $\mathrm{LXA}_{4}(\mathrm{C})$ in timecourse of culture. Skin explants treated with PMA-free oel formulation served as control. Data shown as mean \pm SEM from 3 different young and old donors.
B

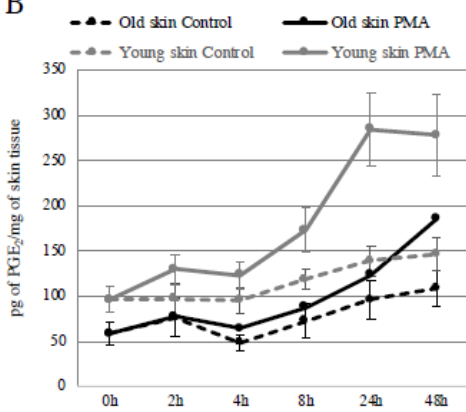

C

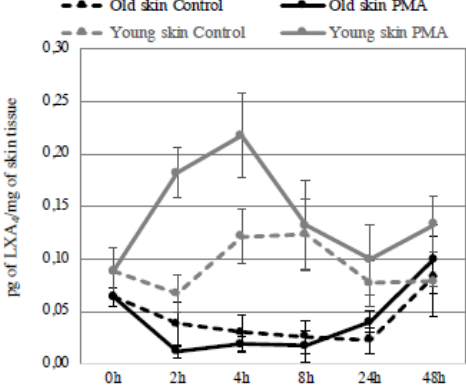

Indeed, we showed that production of PGE2 (Figure 1B) and LXA4 (Figure 1C) were early, intense and temporally regulated in PMAyoung skin. In contrast, there was weaker delayed inflammatory response with seemingly defective LXA4 production in PMA-old skin. Production of LXA4 and PGE2 respectively peaked at 4 hours and 24 hours in young skin, may be due to the persistence of PMA that has half-life estimated at about 24 hours [8]. Nevertheless, production of PGE2 started to decrease at 24 hours, suggesting that LXA4 had induced a stop signal to end inflammation. Conversely, production of PGE2 increasingly increased over time in old skin as if proresolution pathways were compromised.

Hence, metabololipidomic profiling suggests that the AA pathway drives inflammation with early endogenous resolution programs (AA-derived SPMs) in young skin. Skin aging may involve an endogenous inflammation resolution program displaying dysregulation and/or absence of proresolving mediators. Taken together, the present results suggest a role for lipoxins to rebalance cutaneous inflammation during aging and rescue failed resolution in aged skin.

\section{Acknowledgment}

This work was supported by the Gattefossé company (Saint- Priest, France), specialists in lipid chemistry and plant extraction for the health and beauty industries.

\section{References}

1. Kendall AC, Nicolaou A. Bioactive lipid mediators in skin inflammation and immunity. Prog Lipid Res. 2013; 52: 141164.

2. Kendall AC, Pilkington SM, Massey KA, et al. Distribution of bioactive lipid mediators in human skin. J Invest Dermatol. 2015; 135: 1510-1520.

3. Norris PC, Serhan CN. Metabololipidomic profiling of functional immunoresolvent clusters and eicosanoids in mammalian tissues. Biochem Biophys Res Comm. 2018; 504: 553-561.

4. Fullerton JN, O'Brien AJ, Gilroy DW. Pathways mediating resolution of inflammation when enough is too much. J Pathol. 2013; 231: 8-20.

5. Shaw AC, Goldstein DR, Montgomery RR. Age-dependent dysregulation of innate immunity. Nat Rev Immunol. 2013; 13: 875-887.

6. Arnardottir HH, Dalli J, Colas RA, et al. Aging delays resolution of acute inflammation in mice reprogramming the host response with novel nano-proresolving medicines. J Immunol. 2014; 193: 4235-4244.

7. Le Faouder P, Baillif V, Spreadbury I, et al. LC-MS/MS method for rapid and concomitant quantification of proinflammatory and pro-resolving polyunsaturated fatty acid metabolites. J Chromatogr B, Analyt Technol Biomed Life Sci. 2013; 932: 123-133.

8. Risks for human and animal health related to the presence of phorbol esters in Jatropha kernel meal. EFSA J. 2015; 13: 4321.

\section{(c) 2019 Lopez-Gaydon A, et al. This article is distributed under the terms of the Creative Commons Attribution 4.0 International License}

